Pacific Journal of Mathematics

CROSS-SECTIONS OF DECOMPOSITIONS 


\title{
CROSS-SECTIONS OF DECOMPOSITIONS
}

\author{
J. P. RILEY
}

The following question was raised by $R$. H. Bing: "Is it true that if $G$ is a monotone decomposition of $E^{3}$ into straight line intervals and one-point sets, then $E^{3} / G$ is homeomorphic to $E^{3}$ ?" In his paper "Point-like decompositions of $E^{3}$ " he described a possible counter example. This example has the interesting property that it has many tame cross-sections, but if its decomposition space is homeomorphic to $E^{3}$, its set of nondegenerate elements would have to form a wild Cantor set. This suggests that it would be interesting to study the connection between the embedding of a cross-section and the embedding of the set of nondegenerate elements in the decomposition space.

1. Introduction. Most of the terminology and notation used in this paper is standard. The reader is referred to [1], [3], [4], and [6].

If $S$ is a 2-sphere in $E^{3}$, then by Int $S$ we will mean the bounded component of $E^{3}-S$ and by Ext $S$, the unbounded component.

Let $G$ be an upper semi-continuous decomposition of $E^{3}$ and let $H$ be the set of all nondegenerate elements of $G$. We will say that a set $R \subset E^{3}$ is a cross-section of $G$ if (i) $R \cap h$ is a singleton for each $h \in H$, and (ii) the natural map $P$ restricted to $R$ is homeomorphism onto $\overline{P(H)}$. We note that cross-sections exist only for certain decompositions. A simple example may be constructed as follows: Let $a_{n}=$ $1 / n$, for $n=1,2, \cdots$ and let $b_{n}=-1 / n$ for $n=1,2, \cdots$. Let the set of nondegenerate elements of our decomposition consist of the closed interval from $(0,1,0)$ to $(0,-1,0)$, the closed interval from $\left(a_{n}\right.$, $1 / 2,0)$ to $\left(a_{n}, 1,0\right)$ for each positive integer $n$, and the closed interval from $\left(b_{n},-1 / 2,0\right)$ to $\left(b_{n},-1,0\right)$ for each positive integer $n$.

II. Cross-sections of decompositions. The following question naturally arises: How are the embeddings of a cross-section $R$ and $\overline{P(H)}$ related when $E^{3} / G$ is homeomorphic to $E^{3}$ ? We will give some partial results to this question.

THEOREM 1. Let $G$ be an upper semi-continuous decomposition of $E^{3}$ into points and straight line intervals pointing in only a countable number of directions whose lengths are bounded away from zero such that $P(H)$ is a compact 0-dimensional set. If there exists a crosssection $C$ of $G$ then $C$ is tame.

Proof. In the special case where the elements of $H$ point in only 
one direction, we can easily show the tameness by a modification of the proof of Theorem 2 of [7].

Suppose that $H=\bigcup_{n=1}^{\infty} H_{n}$ where the elements of $H_{n}$ are all parallel and if $h_{1} \in H_{i}$ and $h_{2} \in H_{j}$ where $j \neq i$ then $h_{1}$ is not parallel to $h_{2}$. Let $C_{n}$ be the set of all points $c \in C$ such that $c \in h$ for some $h \in H_{n}$. Let $G_{n}$ be the upper semi-continuous decomposition of $E^{3}$ whose only nondegenerate elements are the elements of $H_{n}$ and let $P_{n}$ be the natural map. Then $E^{3} / G_{n}$ is homeomorphic to $E^{3}$ and $P_{n}\left(H_{n}\right)$ is tame in $E^{3} / G_{n}$. So by the special case $C_{n}$ is tame and by Corollary 2 to Theorem 3 of [7], $C$ is tame.

The following two lemmas will be stated without proof. Their proofs are similar to that of Lemma $A$ of [7] and use standard techniques. Lemma B is similar to Theorem 2.3 of [3].

Lemma A. Let $G$ be an upper semi-continuous decomposition of $E^{3}$ such that $P(H)$ is a compact 0-dimensional set. Let $h \in H$ and suppose that there exist 2-spheres $S_{1}$ and $S_{2}$ such that $h \subset \operatorname{Int} S_{1} \cap \operatorname{Int} S_{2}$ and $\left(S_{1} \cup S_{2}\right) \cap(\cup H)=\varnothing$. Then there exists a 2-spheres $S$ such that $h \subset \operatorname{Int} S, S \cup \operatorname{Int} S \subset S_{1} \cup \operatorname{Int} S_{1}$, and if $k \in H$ then $k \subset \operatorname{Int} S$ iff $k \subset$ Int $S_{1} \cap \operatorname{Int} S_{2}$.

Lemma B. Let $S_{1}, S_{2}, \cdots, S_{n}$ be a finite collection of 2-sphere whose interiors cover $\cup H$ and which miss $\cup H$. Then there exists a finite collection of 2-spheres $R_{1}, R_{2}, \cdots, R_{n}$ such that $R_{1}=S_{1},\left(R_{i} \cup\right.$ Int $\left.R_{i}\right) \cap\left(R_{j} \cup \operatorname{Int} R_{j}\right)=\varnothing$ if $i \neq j$, and $h \subset \operatorname{Int} R_{i}$ iff $h \subset \operatorname{Int} S_{i}$ and $h \cap$ Int $S_{j}=\varnothing$ for $j<i$.

Theorem 2. Let $C$ be a wild Cantor set in $E^{3}$ with the property that if $x$ and $y$ are distinct points of $C$, then there exist disjoint 2spheres $S_{1}$ and $S_{2}$ such that $\left(S_{1} \cup S_{2}\right) \cap C=\varnothing, x \in \operatorname{Int} S_{1} \cap$ Ext $S_{2}$ and $y \in \operatorname{Int} S_{2} \cap$ Ext $S_{1}$. Then there exists a monotone decomposition $G$ of $E^{3}$ such that $C$ is a cross-section for $G, E^{3} / G$ is homeomorphic to $E^{3}$ and $P(\bar{H})$ is tame.

Proof. Let $C$ be a wild Cantor set in $E^{3}$ with the required property. For each $x \in C$ we choose a 2 -sphere $S_{1}(x)$ as follows:

Let $N_{1}(x)$ be a 2 -sphere of radius $1 / 2$, centered at $x$. Let $C_{1}(x)=$ $\left\{t \in C \mid t \notin \operatorname{Int} N_{1}(x)\right\}$. Then for each $y \in C_{1}(x)$ choose disjoint 2-spheres $S(y)$ and $R(y)$ such that $(S(y) \cup R(y)) \cap C=\varnothing, x \in \operatorname{Int} S(y) \cap \operatorname{Ext} R(y)$, and $y \in \operatorname{Int} R(y) \cap \operatorname{Ext} S(y)$. Now choose a set $y_{1}, y_{2}, \cdots, y_{n}$ of elements of $C_{1}(x)$ such that $\left\{\right.$ Int $R\left(y_{1}\right)$, Int $R\left(y_{2}\right), \cdots$, Int $\left.R\left(y_{n}\right)\right\}$ covers $C_{1}(x)$. We now apply Lemma $\mathrm{A}$ to get a 2 -sphere $S_{1}(x)$ such that $x \in \operatorname{Int} S_{1}(x)$, $S_{1}(x) \cap C=\varnothing, C_{1}(x) \subset \operatorname{Ext} S_{1}(x)$ and $S_{1}(x) \subset S\left(y_{i}\right) \cup \operatorname{Int} S\left(y_{i}\right)$ for $i=1$, $2, \cdots, n$. Therefore, there exists a finite collection of points $x_{1}, x_{2}, \cdots$, 
$x_{m(1)}$ of $C$ such that $C \subset \operatorname{Int} S_{1}\left(x_{1}\right) \cup \operatorname{Int} S_{1}\left(x_{2}\right) \cup \cdots \cup \operatorname{Int} S_{1}\left(x_{m(1)}\right) . \quad$ We replace $\mathscr{S}_{1}=\left\{S_{1}\left(x_{1}\right), S_{1}\left(x_{2}\right), \cdots, S_{1}\left(x_{m(1)}\right)\right\}$ by another collection of 2spheres $\mathscr{T}_{1}=\left\{T_{11}, T_{12}, \cdots, T_{1 n(1)}\right\}$ satisfying the conclusions of Lemma B with respect to $\mathscr{S}_{1}$.

We will now proceed to construct a sequence $\mathscr{T}_{1}, \mathscr{T}_{2}, \mathscr{T}_{3}, \cdots$ of finite covers of $C$. Suppose that $\mathscr{T}_{k-1}$ has been chosen. For each point $x \in C$ we choose a 2 -sphere $N_{k}(x)$ centered at $x$ with radius $1 / 2^{k}$. We then proceed to choose $\mathscr{T}_{k}$ by the same process as in the construction of $\mathscr{T}_{1}$. We note that if $y_{1}, y_{2} \in T_{k j} \cap C$ then $d\left(y_{1}, y_{2}\right)<1 / 2^{k-1}$ since $T_{j_{k}} \cap C \subset N_{k}(x)$ for some $x \in C$. Now for $x \in C$ we define $h_{x}$ to be $\bigcap_{k=1}^{\infty}\left(T_{k i} \cup \operatorname{Int} T_{k i}\right)$ where $T_{k i}$ is the 2 -sphere in $T_{k}$ whose interior contains $x$. Let $G$ be the decomposition of $E^{3}$ whose only nondegenerate elements are the nondegenerate elements of $\left\{h_{x} \mid x \in C\right\}$. It follows easily that $G$ is upper semi-continuous and it is clear that $C$ is a cross-section for $G$. A theorem of Harrold [5] shows that $E^{3} / G$ is homeomorphic to $E^{3}$ and from the criteria of [3], we see that $\overline{P(H)}$ is tame.

The Cantor set constructed in [2] is an example of a wild Cantor set satisfying the hypothesis of Theorem 2 .

We can note that if $C$ is a wild Cantor set in $E^{3}$ which does not satisfy the condition of Theorem 2, also, if $C$ is a cross-section of a decomposition $G$ whose decomposition space is homeomorphic to $E^{3}$ then $P\left(H_{G}\right)$ is a wild Cantor set which does not satisfy the condition of Theorem 2.

\section{REFERENCES}

1. S. Armentrout, Monotone decompositions of $E^{3}$, Topology Seminar Wisconsin, 1965, Princeton (1966), 1-25.

2. R. H. Bing, A homeomorphism between the 3-sphere and the sum of two solid horned spheres, Annals of Mathematics, 56 (1952), 354-362.

3. - , Tame Cantor sets in $E^{3}$, Pacific J. Math., 11 (1961), 435-446.

4. Decompositions of $E^{3}$, Topology of 3-manifolds and Related Topics, Prentice Hall (1962), 5-21.

5. O. G. Harrold, Jr., A sufficient condition that a monotone image of the three-sphere be a topological three-sphere, Proc. Amer. Math. Soc., 9 (1958), 846-850.

6. R. L. Moore, Foundations of Point Set Theory, (new ed.), Amer. Math. Soc. Colloquium Publications, 13 (1962).

7. J. P. Riley, Decompositions of $E^{3}$ and the tameness of their sets of nondegenerate elements, Duke Math. J., 38 No. 2, (June, 1971).

Received May 16, 1972 and in revised form September 31, 1972.

UNIVERSITy OF DELAWARE 



\section{PACIFIC JOURNAL OF MATHEMATICS}

\section{EDITORS}

RICHARD ARENS (Managing Editor) University of California

Los Angeles, California 90024

R. A. Beaumon'T

University of Washington Seattle, Washington 98105
J. Dugundj1*

Department of Mathematics University of Southern California Los Angeles, California 90007

D. Gilbarg and J. Milgram Stanford University Stanford, California 94305

\section{ASSOCIATE EDITORS}

E. F. BECKENBACH

B. H. NeUMANN

F. WOLF

K. YOSHIDA

\section{SUPPORTING INSTITUTIONS}

UNIVERSITY OF BRITISH COLUMBIA CALIFORNIA INSTITUTE OF TECHNOLOGY UNIVERSITY OF CALIFORNIA MONTANA STATE UNIVERSITY UNIVERSITY OF NEVADA NEW MEXICO STATE UNIVERSITY OREGON STATE UNIVERSITY UNIVERSITY OF OREGON OSAKA UNIVERSITY

\section{UNIVERSITY OF SOUTHERN CALIFORNIA STANFORD UNIVERSITY UNIVERSITY OF TOKYO UNIVERSITY OF UTAH WASHINGTON STATE UNIVERSITY UNIVERSITY OF WASHINGTON AMERICAN MATHEMATICAL SOCIETY NAVAL WEAPONS CENTER}

* C. R. DePrima California Institute of Technology, Pasadena, CA 91109, will replace J. Dugundji until August 1974. 


\section{Pacific Journal of Mathematics}

\section{Vol. 49, No. $1 \quad$ May, 1973}

A. Bigard, Free lattice-ordered modules ...........................

Richard Bolstein and Warren R. Wogen, Subnormal operators in strictly cyclic

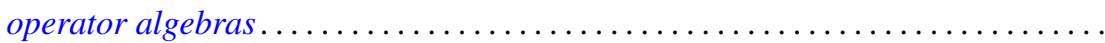

Herbert Busemann and Donald E. Glassco, II, Irreducible sums of simple

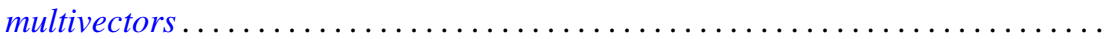

W. Wistar (William) Comfort and Victor Harold Saks, Countably compact groups

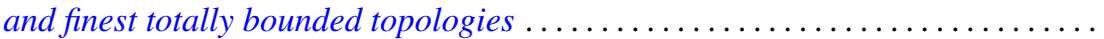

Mary Rodriguez Embry, Maximal invariant subspaces of strictly cyclic operator

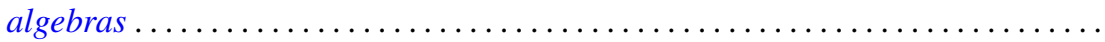

Ralph S. Freese and James Bryant Nation, Congruence lattices of semilattices......

Ervin Fried and George Grätzer, A nonassociative extension of the class of

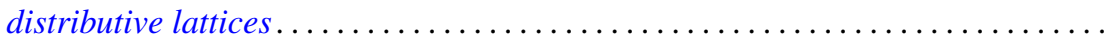

John R. Giles and Donald Otto Koehler, On numerical ranges of elements of locally

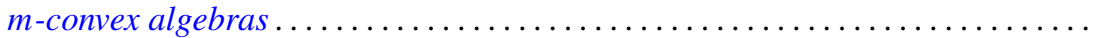

David A. Hill, On dominant and codominant dimension of $\mathrm{QF}-3$ rings ........ John Sollion Hsia and Robert Paul Johnson, Round and Pfister forms over $R(t) \ldots$ I. Martin (Irving) Isaacs, Equally partitioned groups . . . . . . . . . . . . . .

Athanassios G. Kartsatos and Edward Barry Saff, Hyperpolynomial approximation of solutions of nonlinear integro-differential equations.

Shin'ichi Kinoshita, On elementary ideals of $\theta$-curves in the 3-sphere and 2-links in the 4-sphere

Ronald Brian Kirk, Convergence of Baire measures

R. J. Knill, The Seifert and Van Kampen theorem via regular covering spaces ..

Amos A. Kovacs, Homomorphisms of matrix rings into matrix rings ..

Young K. Kwon, HD-minimal but no $H D$-minimal ..........

Makoto Maejima, On the renewal function when some of the mean renewal lifetimes are infinite

Juan José Martínez, Cohomological dimension of discrete modules over profinite groups.

W. K. Nicholson, Semiperfect rings with abelian group of units

Louis Jackson Ratliff, Jr., Three theorems on imbedded prime divisors of principal ideals.

Billy E. Rhoades and Albert Wilansky, Some commutants in $B(c)$ which are almost matrices

John Philip Riley Jr., Cross-sections of decompositions . . .

Keith Duncan Stroyan, A characterization of the Mackey uniformity $m\left(L^{\infty}, L^{1}\right)$ for

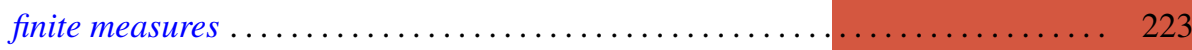

Edward G. Thurber, The Scholz-Brauer problem on addition chains . . . . . . . . . 229

Joze Vrabec, Submanifolds of acyclic 3-manifolds ............

Philip William Walker, Adjoint boundary value problems for compactified singular

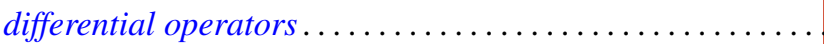

